\title{
PREFACE
}

\section{The First Attempt for Accounts of Environmental Chemistry and Technology Research}

\author{
Jinyong Liu $(\bowtie)^{1}$, Yin Wang $(\bowtie)^{2}$, Haoran Wei $(\bowtie)^{3,4}$ \\ 1 Department of Chemical and Environmental Engineering, University of California, Riverside, Riverside, CA 92521, USA \\ 2 Department of Civil and Environmental Engineering, University of Wisconsin-Milwaukee, Milwaukee, WI 53201, USA \\ 3 Environmental Chemistry and Technology Program, University of Wisconsin-Madison, Madison, WI 53706, USA \\ 4 Department of Civil and Environmental Engineering, University of Wisconsin-Madison, Madison, WI 53706, USA
}

(C) Higher Education Press 2020

In the field of environmental chemical science and technology, researchers have been publishing high-quality research articles on a specific discovery as well as comprehensive reviews on a broader area. After multiple years of research and publication of individual reports, they are looking for a new venue for scientific communication, which will not only summarize a series of their past achievements but also further extend the discussion without including a comprehensive review of $200+$ articles.

During the preparation of individual research reports, researchers oftentimes publish the data that are the most pertinent to the scientific story and the most compelling to highlight the new concepts. Some very interesting data have been considered "off-topic" and excluded from the published work because a reasonable explanation has not yet been available, or those data might distract the reviewers and readers from the main points of work. However, after years, researchers may find them important for reorganizing the research story. As more knowledge has been obtained, some "off-topic" data (and even failed works) that were saved earlier may show novel mechanistic insights, but those data may not be sufficient for a standalone report. Under such circumstances, a mini-review can be an excellent option for researchers to (1) share the story in a more attractive and concise rationale, (2) extend the results and discussions, and (3) express novel opinions.

Inspired by this need from many researchers, this Special Issue focuses on concise account articles describing recent research developments in the chemistry, technology, and process for environmental pollutant removal. In this first attempt, we invited 15 contributions, many of which were from junior researchers. As guest editors, we are grateful to receive these high-quality submissions and enjoy the experience of handling the manuscripts from initial submission to final publication. We appreciate the encouraging support from Prof. Hui Wang, the Editorial Board Member of FESE, on the initial idea of this Special Issue. We thank Dr. Jiao Zhang and Ms. Xiangyi Zhang in the Editorial Office, who provided a tremendous amount of help on this issue. We thank the reviewers for their precious time and efforts. We hope that readers will find these research accounts helpful for both fundamental study and engineering practice. Comments and suggestions are welcome for the Journal to decide whether and when to have the next Special Issue of this style.

Received August 24, 2020

E-mails: jyliu@engr.ucr.edu (J. Liu); wang292@uwm.edu (Y. Wang); haoran.wei3@wisc.edu (H. Wei) 